\title{
Inhibitory Effect of Histone Deacetylase Inhibitor on the Proliferation of Leukemia Cells and Its Anti-Tumor Pharmacology
}

\author{
Shubo Wang \\ College of Pharmacy, Jinan University, Guangzhou, China \\ Email: o_kihiro@163.com
}

How to cite this paper: Wang, S.B. (2021) Inhibitory Effect of Histone Deacetylase Inhibitor on the Proliferation of Leukemia Cells and Its Anti-Tumor Pharmacology. Journal of Biosciences and Medicines, 9, $30-40$.

https://doi.org/10.4236/jbm.2021.91003

Received: November 30, 2020

Accepted: January 15, 2021

Published: January 18, 2021

Copyright $\odot 2021$ by author(s) and Scientific Research Publishing Inc. This work is licensed under the Creative Commons Attribution International License (CC BY 4.0).

http://creativecommons.org/licenses/by/4.0/ (c) (i) Open Access

\begin{abstract}
The aim of this study was to explore the inhibitory effect of histone deacetylase inhibitor (HDACI) on the proliferation of leukemia cells. The two kinds of leukemia cells (human promyelocytic leukemia cell (HL-60) and human acute myelogenous leukemia cell (KG-1)) were selected for in vitro research. Besides, Chidamide, a kind of benzamide HDACI, was applied to induce and culture the HL-60 and KG-1 cells, and the anti-tumor cell proliferation activity of Chidamide on HL-60 and KG-1 was detected by the methyl thiazolyl tetrazolium (MTT) assay, which was 5.6 and 6.1 in turn. The cell scratch experiment verified that Chidamide had the metastasis inhibitory effect on HL-60 and KG-1 cells. Flow cytometry was employed to measure the percentage of apoptotic cells, and it was found that the percentage of apoptotic cells was $55.6 \% \pm 1 \%$ and $48.6 \% \pm 1 \%$ in sequence after HL-60 and KG-1 cells were treated with Chidamide for 36 hours. The number of auto-phagosomes was determined by transmission electron microscopy showing that the number of auto-phagosomes in HL-60 and KG-1 cells was $12 \pm 1$ and $10 \pm 1$, respectively after the induction process of Chidamide. The phosphorylated histone $\mathrm{H} 2 \mathrm{AX}$ protein $(\gamma-\mathrm{H} 2 \mathrm{AX})$ recognition antibody immunofluorescence method was adopted to determine the deoxyribonucleic acid (DNA) damage, and the positive rates of HL-60 and KG-1 cells reached $28.41 \%$ and $26.35 \%$, respectively after Chidamide treatment. Therefore, Chidamide, as a kind of HDACI, could effectively inhibit the proliferation of leukemia cells, so that the results of this experiment had a good guiding meaning for the clinical diagnosis and treatment of leukemia.
\end{abstract}

\section{Keywords}

Tumor Cell Proliferation, Histone Deacetylase Inhibitor, Chidamide, Leukemia Treatment 


\section{Introduction}

At present, malignant tumors have become the number one killer of human health and one of the main causes of human death [1]. As a malignant tumor, leukemia has seriously affected the survival and health of human beings. In traditional tumor treatment methods, the general method is to directly intervene in tumor cell mitosis and the synthesis and repair of DNA [2]. However, this treatment method is often accompanied by serious side effects, not only affecting the clinical treatment effect but also leading to a great burden on the patient's body.

Studies have pointed out that histone acetylation and histone de-acetylation (HDAC) are widespread in collective growth and development, and the level of histone acetylation is closely related to tumor growth [3]. The imbalance between histone acetylation and HDAC results in abnormal differentiation and proliferation of cells, so as to cause the production of malignant tumors in the body. A large number of experiments have proved that the level of HDAC is often overexpressed in tumor cells [4], and the overexpression of HDAC shows a positive correlation with tumor invasion. Therefore, some specific drugs were employed to inhibit HDAC in the body in order to suppress the tumor expression, which has become a new clinical treatment for tumors. The basic mechanism of HDACI in the treatment of tumors is that the specific functions and groups in HDACI inhibit the structure of zinc finger $\left(\mathrm{Zn}^{2+}\right)$ in HDAC [5]. In terms of cell performance, HDACI mainly manifests itself as inhibiting tumor cell growth, metastasis, and invasion, blocking the cell cycle, and inducing apoptosis [6], but the specific medicinal functions still need a lot of research data to prove.

In this study, Chidamide (a type of benzamide HDACI) was applied to investigate the inhibitory effect of leukemia cells. HL-60 and KG-1 cells were selected for in vitro experiments to explore the mechanism of Chidamide on leukemia cells. Moreover, the objective of this study was to provide a theoretical basis for the clinical diagnosis of leukemia.

\section{Literature Review}

\subsection{Research Progress on HDACI-Induced Cell Apoptosis}

Studies have shown that high expression of HDAC suppresses the tumor cell apoptosis, while HDACI induces the tumor cell apoptosis through transcription [7]. This process is often accompanied by major changes in gene expression and chromatin structure. Apoptosis includes 2 pathways (endogenous and exogenous pathways). The endogenous pathway is also called the mitochondrial apoptosis pathway [8]. It means that the apoptosis pathway is activated to release the apoptotic proteins into cytoplasm when tumor cells are stimulated by signals (such as chemotherapy drugs), and the pro-apoptotic proteins are combined with apoptotic protease activating factor-1 (APAF-1) in the cell to activate the downstream Caspase-9, so as to induce cell apoptosis. For example, MHY218 (a type of hydroxamic acid HDACI) can induce the down-regulation of B-cell 
lymphoma-2 (BCL-2) and the up-regulation of BCL2-associated X (BAX) expression in colon cancer cells. It directly leads to a marked increase in the ratio of BAX/BCL-2 under the guidance of a drug concentration-dependent manner. In addition, the activities of Caspase-3, Caspase-8, and Caspase- 9 also rise obviously. The above all proves that MHY218 can promote tumor cell apoptosis through endogenous pathways.

The exogenous pathway of apoptosis is the activation of the apoptosis pathway after the binding of the tumor necrosis factor (TNF) superfamily receptor and the corresponding death ligand [9], so as to cause the activation of Caspase- 8 and Caspase-10. Besides, these two proteases further stimulate the activation of Caspase-3, Caspase-6, and Caspase-7, so that tumor cell apoptosis is eventually induced. Furthermore, HDACI is selective when it induces apoptosis [10] and can't induce apoptosis for normal cells.

\subsection{Research Progress on HDACI-Induced Cell Autophagy}

In addition to cell apoptosis, cell autophagy is also one of the means to control programmed cell death. Autophagy generally occurs in eukaryotic cells, and the effect of autophagy is to recycle its own substances [11]. The way of autophagy is that the organelle or cytoplasm combines with intracellular lysosomes to degrade after it is wrapped by the isolation membrane. Autophagy can also degrade error proteins in cells and rebuild cell structure, which can be regarded as a self-rescue process of cells. The isolation membrane formation of autophagy is controlled by Becin-1 gene. In clinical research, many factors such as different cell types and drug types will influence the different biological functions of autophagy induced by different HDACI monomers, and HDACI can induce the high expression of Becin-1 gene [12]. Clinical studies have indicated that tumor lesions can lead to abnormal autophagy. In many cancerizations, there are deletions in Becin-1 genesto varying degrees, and the down-regulation of Becin-1 gene may increase the chance of cancerization.

\subsection{Research Progress on HDAC Anti-Tumor Angiogenesis and Metastasis}

One of the characteristics of tumor growth is the formation and metastasis of blood vessels in the tumor [13]. Tumor growth is divided into an avascular phase and a vascular phase. In the avascular phase of tumor growth, the oxygen supply mode of diseased cells in the tumor is mainly the diffusion of surrounding tissues. However, the nutrients provided by the surrounding tissues can no longer meet the metabolism of the hyperplastic diseased cells as the tumor grows. The tumor tissues will solve the cell nutrient supply by proliferating blood vessels. At present, there is a large amount of evidence that HDACI has an excellent effect of inhibiting angiogenesis [14]. Studies have displayed that common HDACIs such as tumor-specific antigen (TSA), suberoylanilide hydroxamic acid (SAHA), and romidepsin (FK228) present the strong ability in tumor angioge- 
nesis.

In advanced cancer, it is often accompanied by tumor metastasis. Tumor metastasis is related to a variety of cell behaviors, including blood vessel growth, invasion, and colony formation. Tumor metastasis will affect other normal tissues and organs, making clinical diagnosis difficult to cure. Tumor metastasis is similar to angiogenesis, both of which promote the movement of tumor cells after changing cell adhesion characteristics and regulating the ability of matrix degradation [15]. In clinical cases, the extracellular matrix (ECM) will have to first be degraded to break the barrier between tissues if tumor tissue migration occurs. The acetylation level of histones is directly associated with the aggressiveness of tumor cells. HDACI inhibits the transcription of matrix metalloproteinase (MMP) by up-regulating the expression of RECK inhibitors [16], which decreases the degradation ability of ECM and exhibits a strong anti-migration effect.

\section{Experimental Methods}

The leukemia cell line HL-60 applied in this experiment was donated by Tianjin Institute of Blood, China, and the leukemia cell line KG-1 came from 6 patients with acute myeloid leukemia in Jinan Hospital. This experiment had been authorized by the Ethics Committee of Jinan Hospital. The subjects and their family members had signed the informed consent forms.

\subsection{In Vitro Anti-Leukemia Cell Proliferation Activity Test}

In this study, leukemia cells (HL-60 and KG-1) were selected to test their anti-tumor cell activities in vitro. The specific operation was to inoculate cells at different phases and make them adhere to a 96-well plate. Then, HL-60 and KG-1 cells were cultured with Chidamide compound with concentration of 80 $\mu \mathrm{M}$ for 60 hours. Next, MTT was added into the plate to culture for 6 hours. After the incubation, the MTT blue-violet crystal formazan precipitate was taken and dissolved in dimethyl sulfoxide (DMSO). The absorbance of the sample was measured under a wavelength of $570 \mathrm{~nm}$ with a Spectramax M5 microtiter plate luminometer, and there were multiple equilibrium tests under the same conditions.

\subsection{Activity Test of Leukemia Cell Metastasis in Vitro}

According to related studies, HDAC6 had a great effect on tumor cell metastasis. In this study, there was a Chidamide anti-metastatic activity test, which has an obvious inhibitory effect on HDAC6. The two leukemia cells (HL-60 and KG-1) were used for cell scratch experiments.

\subsection{Detection of Leukemia Cell Apoptosis in Vitro}

HDACI-induced apoptosis is a crucial means to inhibit tumors. In this study, HL-60 and KG-1 cells were inoculated into a 6-well plate, and the number of 
cells was $6 \times 10^{4}$ in each well. The leukemia cells were cultured for 12 hours until they were completely attached to the wall. Then, Chidamide was added into the plate, so as to adjust the concentration to $80 \mu \mathrm{M}(80 \mu \mathrm{M}$ was selected as the most suitable drug concentration for the two cells). Next, they were processed for 12, 24 , and 36 hours. The trypsin with the concentration of $0.25 \%$ was added for digestion. After the digestion, trypsin was aspirated and discarded, and then, the medium was used for pipetting. After blowing and aspirating were finished, there was centrifugation for 6 minutes to collect the cell pellet. Besides, the speed of centrifuge was set to $800 \mathrm{rpm}$. The cell pellet was rinsed with $1 \mathrm{~mL}$ of phosphate buffer saline (PBS), and centrifuged twice at $800 \mathrm{rpm}$ for 6 minutes. 1.5 $\mathrm{mL}$ of $60 \%$ ethanol was added into the centrifugal pellet and mixed uniformly, which was kept at $6^{\circ} \mathrm{C}$ for 12 hours. After incubation, it was centrifuged at 800 rpm for 6 minutes, and the supernatant was discarded. Then, the cell pellet was washed twice with $1.5 \mathrm{~mL}$ of PBS and centrifuged at $800 \mathrm{rpm}$ for 6 minutes. In a dark environment, $600 \mu \mathrm{L}$ of staining solution was added into the pellet to carefully blow and suck, the temperature was controlled at $25^{\circ} \mathrm{C} \pm 2^{\circ} \mathrm{C}$. After $30 \mathrm{mi}-$ nutes of staining, the flow cytometry was applied to detect the percentage of stained cells in the total cells at the G1, S, G2, and M phases in turn.

\subsection{Detection of Leukemia Cell Autophagy in Vitro}

HL-60 and KG-1 cells were inoculated into a 6-well plate, with $6 \times 10^{4}$ cells in each well. They were cultured at $25^{\circ} \mathrm{C} \pm 2^{\circ} \mathrm{C}$ for 8 hours (their coverage of about $70 \%$ ). After the induced treatment of Chidamide with $80 \mu \mathrm{M}$ for 16 hours, the sample was fixed in a glove box for 6 hours with 3\% glutaraldehyde solution prepared by a buffer with $\mathrm{pH}$ of 7 . After rinsing the sample with buffer solution for 3 times, it was fixed with $1.2 \%$ osmium for 10 hours. Then, buffer solution was employed to wash the mixed sample 3 times. Besides, it was dehydrated with $40 \%, 60 \%, 90 \%, 95 \%$, and $100 \%$ alcohol for 30 minutes at different concentrations in sequence. After dehydration, the sample was replaced with propylene epoxide at different concentrations (15\%,30\%, 90\%, 100\% and 100\%) (each concentration for 30 minutes). After the replacement, the cells were soaked and the fixed sections were detected through transmission electron microscopy.

\subsection{Detection of Leukemia Cell Differentiation in Vitro}

A 6-well plate was adopted to culture the HL-60 and KG-1 cells, and there were $6 \times 10^{4}$ cells in each well. The Chidamide of $40 \mu \mathrm{M}, 60 \mu \mathrm{M}, 80 \mu \mathrm{M}$, and $100 \mu \mathrm{M}$ was added to treat the cells. The plate was placed in an incubator with an environment of $35^{\circ} \mathrm{C} \pm 2{ }^{\circ} \mathrm{C}$ and $5 \% \mathrm{CO}_{2}$ for 12,24 , and 36 hours, so as to collect cells. Then, the cells were washed with $15 \mu \mathrm{L}$ of PBS, and the supernatant was discarded. The cell sample was re-suspended with $80 \mu \mathrm{L}$ of PBS and added with $1.5 \mu \mathrm{L}$ of $\mathrm{CD} 11 \mathrm{~b}$ antibody. A control group was set, added with $1.5 \mu \mathrm{L}$ of isotype antibody, and incubated at $5^{\circ} \mathrm{C}$ in dark for 1 hour. After incubation, the sample was washed with $15 \mu \mathrm{L}$ of PBS and centrifuged at $2500 \mathrm{rpm}$ for 6 minutes. After 
centrifugation, it was re-suspended with $240 \mu \mathrm{L}$ of PBS and transferred into a flow tube. Finally, the proportion of $\mathrm{CD}_{11} \mathrm{~b}^{+}$cells in the whole sample was determined by the flow cytometry.

\subsection{Detection of DNA Damage of Leukemia Cells in Vitro}

The fetal bovine serum (FBS) was mixed with dulbecco's modified eagle medium (DMEM) to configure a cell culture medium (volume ratio about 1:9 of FBS and DMEM). HL-60 and KG-1 cells were inoculated in cell culture flask and the prepared cell culture medium was added into the flask, so as to culture in a cell culture tank for 8 hours to observe the degree of cell fusion. When the fusion reached about $85 \%$, the medium was taken and discarded. Then, $80 \mu \mathrm{L}$ of Chidamide digested cells with concentration of $80 \mu \mathrm{M}$ were added, and they should be mixed evenly. HL-60 and KG-1 cells with good growth condition were removed and inoculated in a culture dish for 12 hours. After the culture, $25 \mu \mathrm{L}$ of trypsin was added to prepare cell suspension, so as to process the cells for 1 hour. What's more, it was stored in at $0-5^{\circ} \mathrm{C} .15 \mu \mathrm{L}$ of cell suspension was carefully absorbed to inject into the cell count plate. $15 \mu \mathrm{L}$ of cell suspension was absorbed again and mixed with the pre-melted lipopolysaccharide gel, which was immersed in a slide and was lysed for 1 hour with the addition of $20 \mathrm{~mL}$ of cell lysis buffer. After full lysis, the slide was electrophoresed in a dark place for 30 minutes, washed twice with deionized water, dehydrated in $100 \%$ alcohol for 30 minutes, and dried at room temperature. The slide was stained in dark with SYBR gold dye for 1 hour and dried at room temperature. The stained slide was placed under a fluorescence microscope to observe the comet trailing. Moreover, the $\gamma$-H2AX recognition antibody immunofluorescence assay was employed to detect the positive rate of $\gamma-\mathrm{H} 2 \mathrm{AX}$ focal point in the nucleus.

\section{Results and Discussion}

\subsection{Determination of Cell Proliferative Activity by MTT Assay}

The growth inhibition of Chidamide inhibitor on K562 (a type of human leukemia cell line) was detected by MTT assay. The anti-tumor proliferation activity of Chidamide on HL-60 and KG-1 cells was determined to be 5.6 and 6.1, respectively. It revealed that the membrane permeability of HL-60 and KG-1 molecules was positively affected under the effect of Chidamide, resulting in the high anti-proliferation activity of Chidamide against the two kinds of leukemia cells.

\subsection{Determination of Cell Metastasis Activity by Cell Scratch Test}

Figure 1 showed that the cell metastasis ability of HL-60 and KG-1 cells treated with $80 \mu \mathrm{M}$ and $120 \mu \mathrm{M}$ of Chidamide for 12,24 and 36 hours in turn was obviously lower than the ability of the blank control group $(P<0.05)$. It indicated that Chidamide had marked inhibitory effect on the metastasis of HL-60 and KG-1 leukemia cells. 

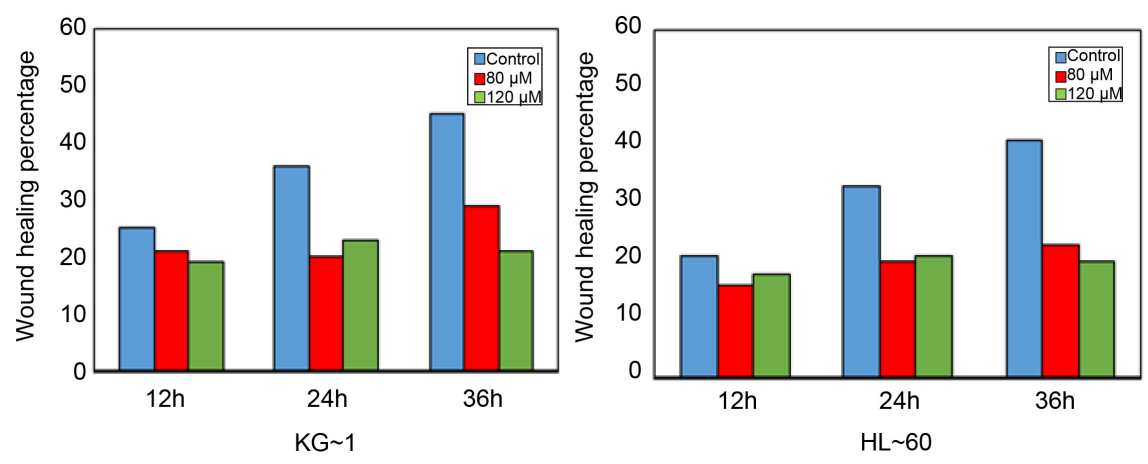

Figure 1. Comparison on the cell metastasis ability of HL-60 and KG-1 cells at different times through the cell scratch experiment.

\subsection{Determination of Cell Apoptosis by Flow Cytometry}

Flow cytometry was adopted to test the cells, and the results were shown in Figure 2 below. After 12 and 24 hours of HL-60 cells processed by Chidamide in sequence, the apoptosis percentage of KG-1 increased but its growth rate was slightly low. After 12 and 24 hours of processing, the apoptosis percentage reached $18.7 \%$ and $31.5 \%$, respectively. Besides, the apoptosis percentage rose sharply to $48.6 \% \pm 1 \% 36$ hours after processing.

Similarly, the percentage of apoptotic cells increased from $4.5 \%$ to $19.3 \%$ and $29.4 \%$, respectively at 12 and 24 hours after treatment with Chidamide on HL-60 cells. Moreover, the percentage of apoptotic cells elevated to $55.6 \% \pm 1 \%$ after 36 hours of treatment. According to the data results, Chidamide had a substantial effect on the apoptosis of HL-60 and KG-1 leukemia cells under the control of different times.

\subsection{Detection of Cell Autophagy by Transmission Electron Microscopy}

The transmission electron microscopy was applied to measure the cells. The results showed that the number of auto-phagosomes after 16 hours of HL-60 and KG-1 cells induced and processed by Chidamide had a great increase in contrast to the number of the blank control group. In addition, auto-phagosomes under the transmission electron microscopy displayed the double membrane structure with embedding cytoplasm. There were 3 auto-phagosomes in the blank control group, and the number of auto-phagosomes was $12 \pm 1$ and $10 \pm 1$ in the HL-60 and KG-1 treatment groups, respectively. The mechanism was speculated that the acidic environment of lysosomes in HL-60 and KG-1 leukemia cells was destroyed after treatment with Chidamide, leading to inhibition of the binding effect of lysosomes and auto-phagosomes. Thus, Chidamide could induce autophagy in HL-60 and KG-1 cells.

\subsection{Determination of Cell Differentiation by Flow Cytometry}

Flow cytometry was applied to determine the cells. Figure 3 indicated that Chidamide markeldy promoted the expression of CD11b on the surface of HL-60 

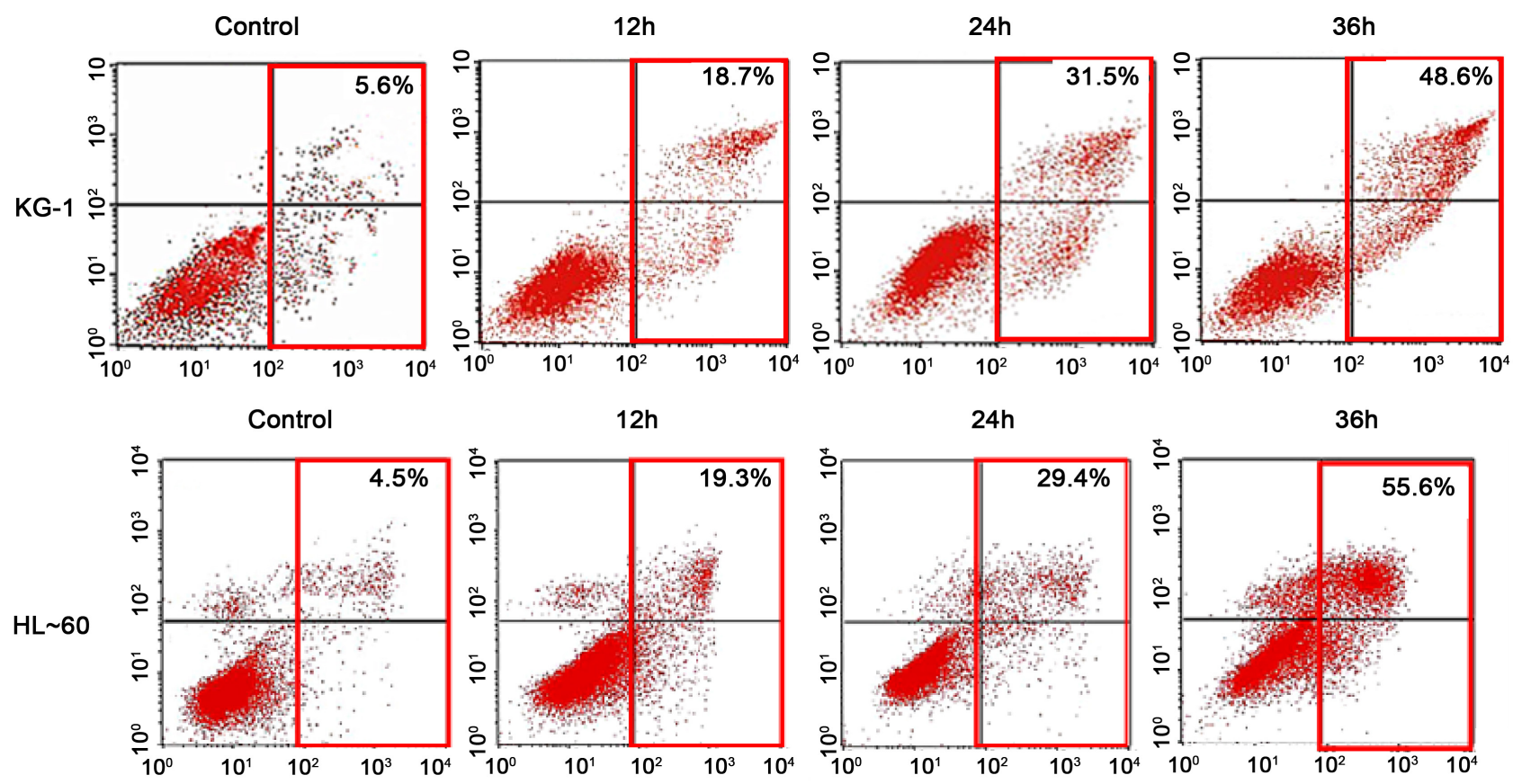

Figure 2. Effects of Chidamide on the percentage of apoptotic cells in KG-1 and HL-60 cells.
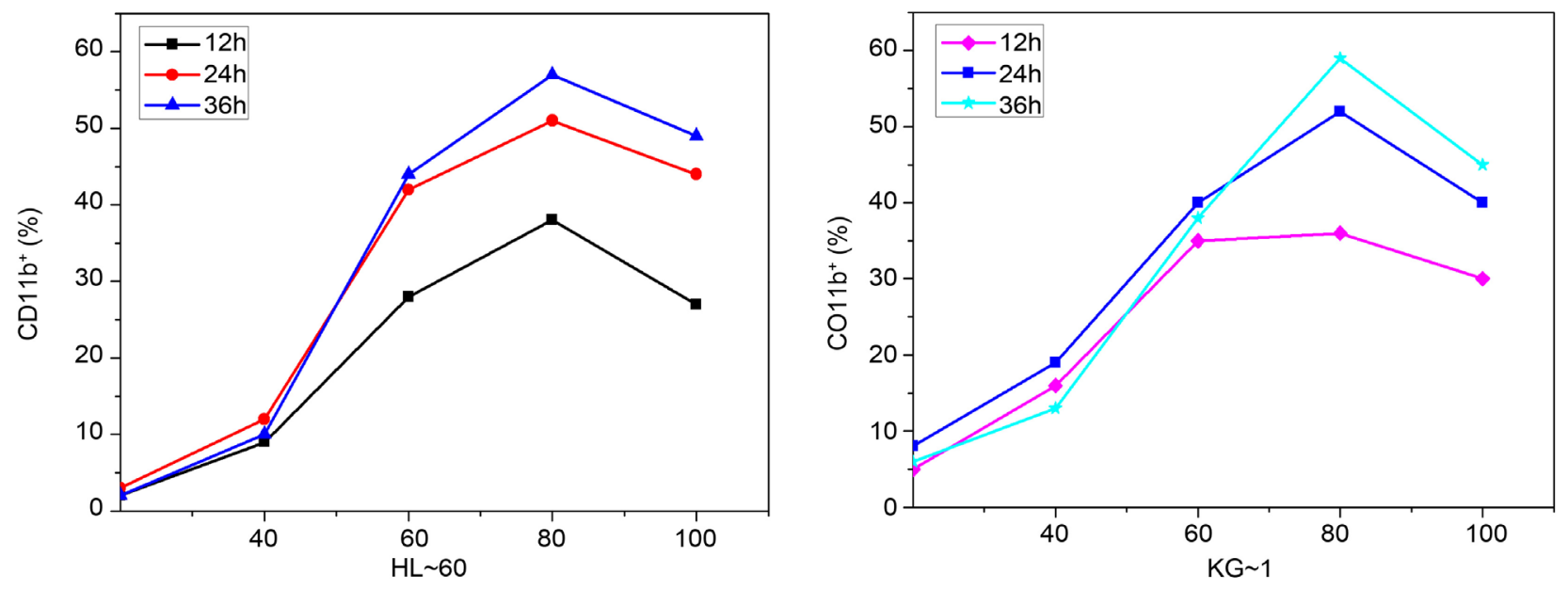

Figure 3. Effects of Chidamide on cell differentiation.

and KG-1 leukemia cells, while CD11b expression in HL-60 and KG-1 cells greatly increased after treatment with Chidamide. Combined with morphological analysis of the cells, it was found that some cells had lobulated nuclei. The above meant that Chidamide was possessed with a strong promoting effect on the differentiation of HL- 60 and KG-1 cells to some extent.

\subsection{Determination of DNA Damage by $\gamma$-H2AX Recognition Antibody Immunofluorescence Assay}

The cells were detected by immunofluorescence assay with $\gamma$-H2AX recognition antibody. The results suggested that HL-60 and KG-1 cells were treated with Chidamide for 12 hours, so as to increase the comet trailing length of both cells 
by $34.2 \%$ and $39.5 \%$, respectively in contrast to the length of the blank control group. The positive rates of HL-60 and KG-1 cells reached $28.41 \%$ and $26.35 \%$ in turn, after the detection by immunofluorescence method with $\gamma$-H2AX recognition antibody. It could indicate that Chidamide could cause the growth of DNA double-strand breaks, and there were a large number of DNA double-strand breaks that were still not repaired. In summary, Chidamide could induce DNA damage in HL-60 and KG-1 cells and inhibit DNA repair.

\section{Conclusions}

In this study, the anti-tumor mechanism of HDACI was deeply explored, so two types of leukemia cells (HL-60 and KG-1) were selected as research objects, and Chidamide was regarded as HDACI for anti-tumor pharmacology research. MTT assay was adopted to detect the growth inhibition of Chidamide on HL-60 and KG-1 leukemia cells, which proved the anti-proliferation activity of Chidamide on HL-60 and KG-1 cells. Cell scratch experiments were conducted to verify the inhibitory effect of Chidamide on HL-60 and KG-1 cells. Flow cytometry was applied to determine the effect of Chidamide on the percentage of apoptotic cells in HL-60 and KG-1 cells, and it was found that Chidamide could enhance the differentiation of HL-60 and KG-1 cells, and had an obvious effect on inducing the apoptosis of HL-60 and KG-1 cells. Transmission electron microscopy was employed to detect the cell autophagy in HL-60 and KG-1 cells, finding that the number of auto-phagosomes increased hugely after being treated with Chidamide. This indicated that there was an autophagy induction effect of Chidamide on HL-60 and KG-1 cells. Finally, $\gamma$-H2AX immunofluorescence assay was applied to detect DNA damage, suggesting that the positive rate of HL-60 and KG-1 cells rose dramatically after treatment with Chidamide.

To sum up, Chidamide could inhibit the cell cycle of HL-60 and KG-1 cells, suppress the metastasis of HL-60 and KG-1 cells, damage the DNA of HL-60 and KG-1 cells, and control DNA repair by inducing apoptosis, differentiation, and autophagy of HL-1 cells. The above had a good guiding effect on the clinical treatment of leukemia, and provided data for the anti-tumor research of HDACI. However, the inhibitory effect of Chidamide on leukemia cells was only proved in vitro, and the experimental results in vivo should be confirmed in the future.

\section{Conflicts of Interest}

The author declares no conflicts of interest regarding the publication of this paper.

\section{References}

[1] White, C., Alshaker, H., Cooper, C., et al. (2016) The Emerging Role of FTY720 (Fingolimod) in Cancer Treatment. Oncotarget, 7, 23106-23127. https://doi.org/10.18632/oncotarget.7145

[2] Williams, D.S., Mouradov, D., Jorissen, R.N., et al. (2019) Lymphocytic Response to Tumour and Deficient DNA Mismatch Repair Identify Subtypes of Stage II/III Co- 
lorectal Cancer Associated with Patient Outcomes. Gut, 68, 465. https://doi.org/10.1136/gutjnl-2017-315664

[3] Hassan, W., Takebayashi, S.I., Abdalla, M.O.A., et al. (2017) Correlation between Histone Acetylation and Expression of Notch1 in Human Lung Carcinoma and Its Possible Role in Combined Small-Cell Lung Carcinoma. Laboratory Investigation, 97, 913-921. https://doi.org/10.1038/labinvest.2017.36

[4] Emmons, M., Rab, R., Sharma, R., et al. (2016) Abstract 294: The Role of the Histone Deacetylase HDAC 8 in Mediating BRAF/MEK Inhibitor Metastatic Dissemination and Resistance. Cancer Research, 76, 294-294. https://doi.org/10.1158/1538-7445.AM2016-294

[5] Sellmer, A., Stangl, H., Beyer, M., et al. (2018) Marbostat-100 Defines a New Class of Potent and Selective Antiinflammatory and Antirheumatic Histone Deacetylase 6 Inhibitors. Journal of Medicinal Chemistry, 61, 3454-3477.

https://doi.org/10.1021/acs.jmedchem.7b01593

[6] Li, X., Peterson, Y.K., Inks, E.S., et al. (2018) Class I HDAC Inhibitors Display Different Antitumor Mechanism in Leukemia and Prostatic Cancer Cells Depending on Their p53 Status. Journal of Medicinal Chemistry, 61, 2589-2603. https://doi.org/10.1021/acs.jmedchem.8b00136

[7] Waibel, M., Vervoort, S.J., Kong, I.Y., et al. (2017) Epigenetic Targeting of Notch1 Driven Transcription Using the HDACi Panobinostat Is a Potential Therapy against T Cell Acute Lymphoblastic Leukemia. Leukemia: Official Journal of the Leukemia Society of America, Leukemia Research Fund, 32, 237-241. https://doi.org/10.1038/leu.2017.282

[8] Zhang, W., Zheng, X., Meng, T., et al. (2016) HDACI Regulates the PI3K/Akt Signaling Pathway to Reverse MCF-7/PTX Resistance by Inhibiting SET. RSC Advances, 6, 48072-48082. https://doi.org/10.1039/C6RA06423J

[9] Lang, I., Füllsack, S., Wyzgol, A., et al. (2015) Binding Studies of TNF Receptor Superfamily (TNFRSF) Receptors on Intact Cells. Journal of Biological Chemistry, 291, 5022-5037. https://doi.org/10.1074/jbc.M115.683946

[10] Sterner, R.M., Kremer, K.N., Amel, D., et al. (2018) Tissue-Nonspecific Alkaline Phosphatase Is Required for MC3T3 Osteoblast-Mediated Protection of Acute Myeloid Leukemia Cells from Apoptosis. The Journal of Immunology, 201, 1086-1096. https://doi.org/10.4049/jimmunol.1800174

[11] GilardiniMontani, M.S., Granato, M., Santoni, C., et al. (2017) Histone Deacetylase Inhibitors VPA and TSA Induce Apoptosis and Autophagy in Pancreatic Cancer Cells. Cellular Oncology, 40, 167-180. https://doi.org/10.1007/s13402-017-0314-Z

[12] Katagiri, H., Nakayama, K., Razia, S., et al. (2015) Loss of Autophagy-Related Protein Beclin 1 May Define Poor Prognosis in Ovarian Clear Cell Carcinomas. International Journal of Oncology, 47, 2037-2044. https://doi.org/10.3892/ijo.2015.3191

[13] Zang, J., Liang, X., Huang, Y., et al. (2018) Discovery of Novel Pazopanib-Based HDAC and VEGFR Dual Inhibitors Targeting Cancer Epigenetics and Angiogenesis Simultaneously. Journal of Medicinal Chemistry, 61, 5304-5322. https://doi.org/10.1021/acs.jmedchem.8b00384

[14] Maiti, A., et al. (2019) Class I Histone Deacetylase Inhibitor Suppresses Vasculogenic Mimicry by Enhancing the Expression of Tumor Suppressor and Anti-Angiogenesis Genes in Aggressive Human TNBC Cells. International Journal of Oncology, 55, 116-130. https://doi.org/10.3892/ijo.2019.4796

[15] Chidambaram, A., Kavya, S.H., Chidambaram, R.K., et al. (2017) Design, Synthesis, and Characterization of $\alpha, \beta$-Unsaturated Carboxylic Acid, and Its Urea Based De- 
rivatives That Explores Novel Epigenetic Modulators in Human Non-Small Cell Lung Cancer A549 Cell Line. Journal of Cellular Physiology, 233, 5293-5309. https://doi.org/10.1002/jcp.26333

[16] Jia, W., Deng, F., Fu, W., et al. (2019) Curcumin Suppresses Wilms' Tumor Metastasis by Inhibiting RECK Methylation. Biomedicine \& Pharmacotherapy, 111, 1204 1212. https://doi.org/10.1016/j.biopha.2018.12.111 